\title{
Ferromagnetic resonance driven by an ac current: a brief review
}

\author{
C. Wang, H. Seinige, and M. Tsoi \\ Department of Physics, University of Texas at Austin, Austin TX 78712, USA \\ E-mail: tsoi@physics.utexac.edu
}

Received November 1, 2012

\begin{abstract}
Excitation of ferromagnetic resonance (FMR) by an ac current has been observed in macroscopic ferromagnetic films for decades and typically relies on the ac Oersted field of the current to drive magnetic moments into precession and classical rectification of ac signals to detect the resonance. Recently, current-driven ferromagnetic resonances have attracted renewed attention with the discovery of the spin-transfer torque (STT) effect due to its potential applications in magnetic memory and microwave technologies. Here STT associated with the ac current is used to drive magnetodynamics on the nanoscale that enables FMR studies in sample volumes smaller by a factor of 1000 compared to conventional resonance techniques. In this paper, we briefly review the basics of STT-FMR technique and the results of various STT-FMR experiments.
\end{abstract}

PACS: 85.75.-d Magnetoelectronics; spintronics: devices exploiting spin polarized transport or integrated magnetic fields;

75.76.+j Spin transport effects;

76.50.+g Ferromagnetic, antiferromagnetic, and ferrimagnetic resonances; spin-wave resonance;

72.25.Pn Current-driven spin pumping.

Keywords: spintronics, spin-transfer torque, ferromagnetic resonance.

\section{Introduction}

Ferromagnetic resonance (FMR) [1-3] is a unique spectroscopic technique to study magnetodynamics in a variety of systems including magnetic nanostructures and spintronic devices. In conventional FMR, the sample is placed in a microwave resonant cavity in the presence of a static magnetic field and the precessional motion of the sample's magnetization is resonantly excited by an applied ac (microwave frequency) magnetic field. Absorption of microwaves can be detected in the neighborhood of the resonance when the applied microwave frequency matches the natural frequency of the magnetization precession [1-5]. Interestingly, instead of detecting small variations in the absorption signal one can simply measure a dc voltage which appears across the sample at resonance when an ac (microwave) current is applied directly through the sample. This voltage is the result of the rectification processes in which the oscillating magnetic moments affect the electric current flow via, e.g., extraordinary Hall effect and/or anisotropic magnetoresistance [6-9]. In addition to greatly simplifying the detection of FMR, the applied ac current can be used as the driving force of FMR via the so-called spin-transfer torque (STT) effect. The new experimental technique of spin transfer torque driven FMR is the focus of this brief review.

The STT effect refers to a novel method to control and manipulate magnetic moments by an electric current [10-12]. For instance, in a spin valve where two magnets are separated by a nonmagnetic spacer, the electric current flowing between the magnets can transfer spin angular momentum from one magnet to another and results in a STT torque on their magnetic moments. The direction of STT torque depends on the polarity of applied current that can be used for reversible switching of magnetic moments in magnetic memory applications [10]. When an ac current is flowing between the magnets, the resulting STT torque also oscillates and thus can be used to drive FMR. The combination of STT with magnetic resonance techniques extends the applicability of FMR to magnetic nanostructures, thanks to the development of sub-micron fabrication and electrical probing techniques [13-15]. The spin transfer torque-driven FMR technique (STT-FMR) enables FMR studies in sample volumes smaller by a factor of 1000 compared to the conventional methods with resonant microwave cavities. Moreover, the electrical FMR detection based on rectification phenomena associated with anisotropic magnetoresistance (AMR) in single-layer structures, giant 
magnetoresistance (GMR) in metallic spin valves and magnetic multilayers, or tunneling magnetoresistance (TMR) in magnetic tunnel junctions provide the means to both excite and probe electrically the dynamical magnetic phenomena on the nanoscale [13-16]. This makes STT-FMR a unique and powerful tool for characterization of magnetodynamics in nanoscale devices.

This review is organized as follows. In Sec. 2, the STT-FMR basics are discussed. In Sec. 3, we review a variety of experimental arrangements and setups used for STT-FMR observations. Sec. 4 summarizes this review about STT-FMR.

\section{Spin transfer torque-ferromagnetic resonance basics}

The driving force of the precession of magnetic moments in a conventional FMR is the oscillating field-torque $\tau_{B}$ exerted by an oscillating rf magnetic field $B_{r f}$ (see Fig. 1(a)). The driving force of the magnetic precession in STT-FMR is the oscillating STT torque $\tau_{S T T}$ associated with an applied ac current $I_{r f}$ (see Fig. 1(b)). We will illustrate the STT effects of current on a simple spin-valve structure where a free magnetic layer $\mathbf{s}$ is separated from a fixed magnet $\mathbf{s}^{*}$ by a nonmagnetic spacer N (Figs. 2(a),(b)) and the fixed layer acts as a perfect spin filter, i.e., electron spins aligned with its magnetic moment are completely transmitted through the layer, while antiparallel spins are completely reflected. When electron current crosses the spin valve from left to right (Fig. 2(a)), electrons transmitted through the polarizer will be polarized along $\mathbf{S}^{*}$ and exert a torque $\tau_{+}$on $\mathbf{s}$ in a direction so as to align $\mathbf{s}$ with $\mathbf{s}^{*}$. When the current crosses the spin valve from right to left (Fig. 2(b)), electrons reflected from the polarizer will be polarized antiparallel to $\mathbf{s}^{*}$ and exert a torque $\tau_{-}$on $\mathbf{s}$ trying to align $\mathbf{s}$ antiparallel with $\mathbf{s}^{*}$. Note that spin relaxation in $\mathrm{N}$, spin filtering by the free layer and higher-order reflections between $\mathbf{s}$ and $\mathbf{s}^{*}$, among other effects, can change the magnitudes of $\tau_{+}$and $\tau_{-}$torques. In macrospin approximation, which treats the free and fixed layers as single magnetic domains, the current-driven dynamics of the free layer can be described by the Landau- (a)

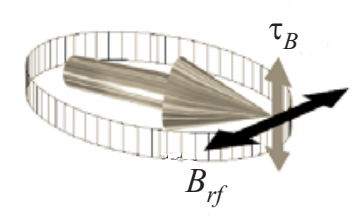

(b)

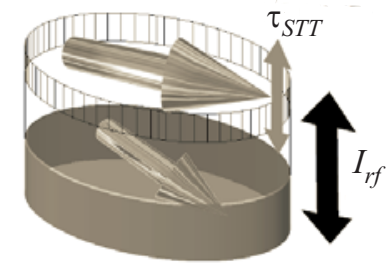

Fig. 1. Driving mechanisms of conventional field-driven FMR (a) and STT-FMR (b). An oscillating magnetic field $\left(B_{r f}\right)$ exerts an oscillating torque $\left(\tau_{B}\right)$ on a magnetic moment (a). An oscillating current $\left(I_{r f}\right)$ which flows between the fixed (bottom) and free (top) layers of a spin valve generates an oscillating STT torque $\left(\tau_{S T T}\right)$ on the magnetic moment of the free layer $(b)$.

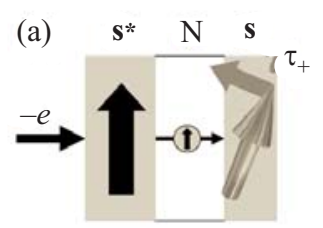

(b) $\quad \mathbf{s}^{*} \mathrm{~N} \quad \mathbf{s}$

(c)
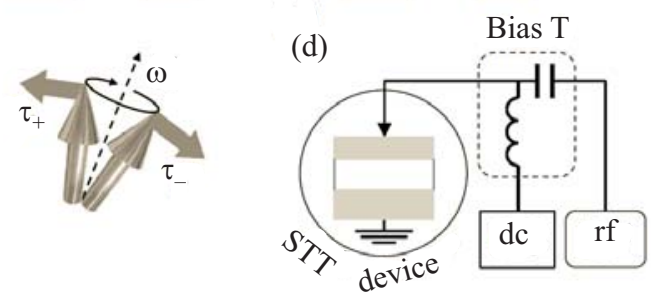

Fig. 2. STT-driven magnetodynamics in a simple spin-valve structure where a free magnetic layer $\mathbf{s}$ is separated from a fixed magnet $\mathbf{s}^{*}$ by a nonmagnetic spacer $\mathrm{N}$ (a), (b). When electron current crosses the spin valve from left to right, electrons transmitted through the fixed polarizer will generate torque $\tau_{+}$on the free layer (a). When the current flows from right to left, electrons reflected from the polarizer will generate torque $\tau_{-}$on the free layer (b). Magnetic precession driven by STT oscillating in sync with the precession (c). Schematic diagram of a STT-FMR experiment with dc and rf electronics connected to a STT device via a bias tee. Both dc and rf currents can be applied to the device and the resulting dc voltage across the device can be measured (d).

Lifshitz-Gilbert (LLG) equation with an addition due to the applied current — STT torque [10]

$$
\tau_{S T T}=\frac{\hbar}{2 e} g j \mathbf{s} \times\left(\mathbf{s}^{*} \times \mathbf{s}\right),
$$

where $j$ is the current density, $g$ is polarization factor, $\mathbf{s}$ and $\mathbf{s}^{*}$ are directions of the free- and fixed-layer magnetizations.

The STT torque (Eq. (1)) depends linearly on the density $j$ of applied current, so a reversal of the current polarity would imply a reversal of the torque. When an ac current is applied to the spin valve, the STT torque oscillates and can drive the magnetic moment of the free layer into resonance if the ac frequency matches the natural frequency of the moment precession. At resonance, the oscillating STT torque acts in sync with the precessional motion of the moment to compensate for the damping losses and continuously drive the precessing moment away from its equilibrium. This mechanism is illustrated in Fig. 2(c) where two moment orientations correspond to two different (opposite) phases of the precessional motion. When the moment is to the left of equilibrium (dashed line in Fig. 2(c)) and the electron current flows from left to right (Fig. 2(a)), the STT torque will tend to increase the precession angle as indicated by the vector $\tau_{+}$in Fig. 2(c). Half a period later, the moment is to the right of equilibrium and $\tau_{-}$will continue increasing the precession due to the reverse current flow (Fig. 2(b)). Such a synchronous variation of the moment and STT torque directions provides a 
means to continuously pump energy from the ac current into the magnetic system.

Note that the in-sync mechanism described above requires a noncollinear configuration of magnetic moments in the two layers. In practice, this is usually achieved by making the free and fixed layers respond differently to an externally applied magnetic field, e.g., by using magnetically soft/hard materials and/or different magnetic anisotropies. In this case, STT-FMR is performed in an applied field well below the saturation field of the multilayer that favors a noncollinear orientation of the two layers. The later is beneficial for a stronger STT [10] but limits the range of magnetic fields (frequencies) for such experiments. STT-FMR experiments at higher frequencies would involve higher magnetic fields and therewith an almost parallel configuration of the two magnetic layers that implies a symmetric precession of the free layer about the fixed layer. In this case, the oscillating STT torque would promote the precession for one half of the period of precession, but oppose it for the other half. This symmetry should be broken so that the ac STT could pump a nonzero net energy into the magnetic system over the precessional period. In real systems, the required asymmetry can come from a number of different sources, including different magnitudes of $\tau_{+}$and $\tau_{-}$torques (see above) or a small non-collinearity of the magnetic moments (e.g., due to sample imperfections).

Figure 2(d) shows a typical setup for STT-FMR experiments. Here a bias tee is used to connect both dc (current source, voltmeter, lock-in amplifier) and rf (microwave generator, oscilloscope) electronics to a STT device (spin valve); various devices will be discussed in the next section. The setup provides a means to apply both ac (micro- wave) and dc currents to the device and measure the resulting voltage. As was discussed earlier, when a microwave current $I(t)=I_{r f} \cos (\omega t)$ at FMR frequency is applied to the device, the oscillating STT can drive the free layer magnetic moment into resonance. The moment precession results in a time-dependent spin-valve resistance due to giant magnetoresistance (GMR) effect as the relative orientation of the free and fixed layers changes continuously. Mixing of the time-dependent component of spin-valve resistance $R(t)=\Delta R \cos (\omega t+\delta)$ with the microwave current $I(t)$ contributes a dc term to the device voltage

$$
V_{\omega}=\frac{1}{2} I_{r f} \Delta R \cos \delta
$$

This rectified voltage develops only at resonance and allows the detection of FMR by simply measuring a dc voltage across the STT device as a function of either the frequency of applied microwave current for a fixed magnetic field, or as a function of the applied magnetic field for a fixed microwave frequency.

Figure 3 shows the results of a typical room-temperature STT-FMR experiment. Figure 3(a) shows the rectified voltage $V_{\omega}$ as a function of applied magnetic field $B$ for different frequencies $\omega / 2 \pi$ of applied rf current in the range from 9-23.7 GHz. The $V_{\omega}(B)$ spectra are shifted vertically for clarity. At higher fields there is a resonance structure (dip) in $V_{\omega}(B)$ which shifts to higher $B s$ at higher frequencies. The resonance frequency vs field relation (solid circles in Fig. 3(b)) is well described by the Kittel's equation [4]

$$
\omega / 2 \pi=\gamma \sqrt{B\left(B+\mu_{0} M_{S}\right)}
$$

with $\mu_{0} M_{S}=0.88 \mathrm{~T}$ for $\mathrm{NiFe}$ (see fit in Fig. 3(b)) that confirms the FMR nature of the observed resonances.
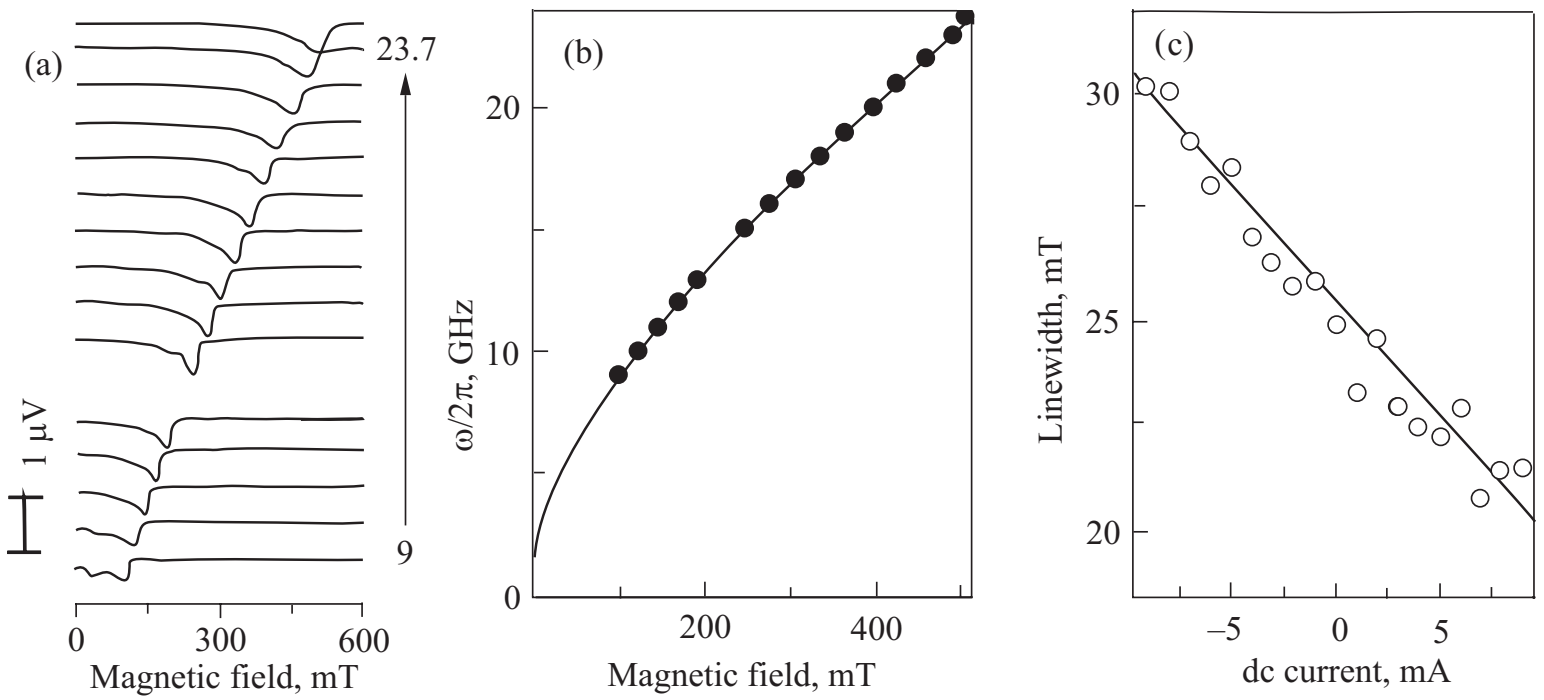

Fig. 3. Typical experimental results of STT-FMR. The rectified dc voltage (at zero dc bias) as a function of applied magnetic field $B$ for different frequencies of applied rf current $\omega / 2 \pi=9,10,11,12,13,15,16,17,18,19,20,21,22,23$, and 23.7 GHz. The spectra are shifted vertically for clarity (a). The experimental resonance frequency vs field relation (solid circles) fitted by the Kittel's equation (b). DC bias dependence of FMR linewidth (c). 
Direct evidence of spin-transfer effect in the observed FMR signals would be the dependence of the resonance linewidth on the dc bias, since the linewidth depends on damping which in turn depends on STT. Since the resonance linewidth is proportional to the effective damping constant which in turn depends linearly on the applied dc current, one can expect a linear dependence of the resonance linewidth on dc current. The dc bias dependence of the FMR linewidth shown in Fig. 3 (c) is consistent with these expectations. A direct outcome from the STT-FMR linewidth analysis is the determination of Gilbert damping in individual nanomagnets with only a million of Bohr magnetons and scalable to even smaller dimensions. The critical dc current of STT-induced dynamics can also be extracted from the linear fit for the bias dependence of the resonance linewidth [14,15].

\section{Spin transfer torque-ferromagnetic resonance experiments}

STT-FMR has been demonstrated in a variety of experiments. In this section, we would like to highlight several pioneering approaches to STT-FMR. Different ways of generating ac STT and probing STT-FMR in magnetic nano-domains are reviewed and discussed.

Magnetic tunnel junctions. The first experimental demonstration of STT-FMR was conducted in 2005 by Tulapurkar et al. [13]. They performed experiments on a PtMn $(15 \mathrm{~nm}) / \mathrm{CoFe}(2.5 \mathrm{~nm}) / \mathrm{Ru} \quad(0.85 \mathrm{~nm}) / \mathrm{CoFeB}$

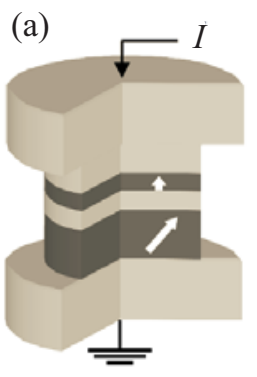

(c)

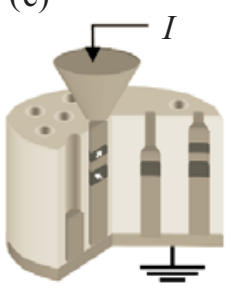

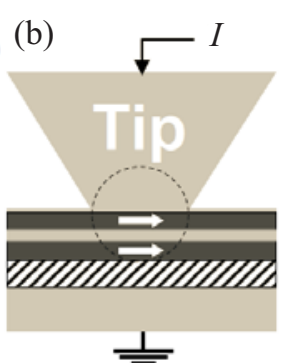

(d)

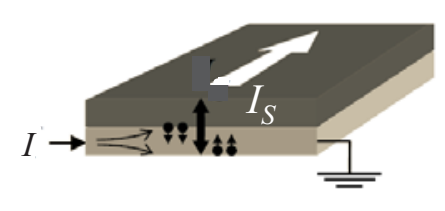

Fig. 4. Examples of devices used in STT-FMR. EBL patterned nanopillars of spin valves (MTJs) with two magnetic - fixed and free - layers (dark grey) separated by a nonmagnetic metallic (insulating) spacer (a). Metallic point-contact devices to EBSV films (b). Magnetic nanowires electrodeposited into porous membrane (c). Ferromagnetic (dark grey)/ Pt (light grey) metal bilayer used in SHE driven FMR experiments. A transverse spin current $I_{S}$ is generated by a longitudinal charge current $I$ in the Pt layer via SHE (d).
$(3 \mathrm{~nm}) / \mathrm{MgO}(0.85 \mathrm{~nm}) / \mathrm{CoFeB}(3 \mathrm{~nm})$ magnetic tunnel junction (MTJ) patterned into oval-shaped pillars of dimension $200 \times 100 \mathrm{~nm}$ (see Fig. 4(a)), using electron-beam lithography and ion milling techniques. Here the bottom synthetic antiferromagnetic $\mathrm{CoFe} / \mathrm{Ru} / \mathrm{CoFeB}$ layer acts as a fixed layer, while the top $\mathrm{CoFeB}$ acts as a free layer. The insulating $\mathrm{MgO}$ spacer serves as a tunneling barrier for the electrons traveling between the free and fixed layers. The dc voltage $V_{\omega}$ produced across the MTJ device was measured as a function of the frequency $\omega$ of the rf current (0.55 mA) applied to MTJ using a setup similar to the one shown in Fig. 2(d). The $V_{\omega}(\omega)$ spectra showed a large resonance structure $(\sim 60 \mu \mathrm{V})$, whose position depends on the magnetic field in good agreement with the Kittel's equation (see Fig. 3 and Eq. (3)).

Further studies of the ac-driven magnetodynamics in MTJs focused on the dependence of spin torque (torkance) on the dc voltage bias. It turns out that, in MTJs under high bias, there is a nonnegligible contribution to STT torque which is perpendicular to the plane formed by the fixedand free-layer magnetic moments, also known as the "field-like" torque. The STT-FMR technique provided a means to study the bias dependence of STT torkances in great detail $[17,18]$.

Metallic nanopillars. Soon after the first demonstration with MTJs, STT-FMR was observed in metallic spin-valve nanopillars [14]. Py (20 nm)/Cu (12 nm)/ $\mathrm{PyCu}(5.5 \mathrm{~nm})$ spin valves were patterned into oval-shaped pillars (see Fig. 4(a)) of dimension $90 \times 30 \mathrm{~nm}$, using electron-beam lithography and ion milling techniques. Various modes of magnetic excitations in both the free (PyCu) and the fixed (Py) layers were found and the signals with the lowest frequency and largest amplitude were attributed to spatially uniform precession (FMR) modes in the two layers. The linear dc bias dependence of the resonance linewidth (see Fig. 3(c)) has confirmed the STT nature of the resonances.

STT-FMR was also observed in spin-valve nanopillars with perpendicular magnetic anisotropy (PMA) which can be used to achieve a non-collinear configuration of the free and fixed layers [19]. For instance, a free [Co $(0.4 \mathrm{~nm}) / \mathrm{Ni}$ $(0.8 \mathrm{~nm})] \times 3$ synthetic PMA layer was used in combination with a fixed $\mathrm{Co}(12 \mathrm{~nm})$ layer and a $\mathrm{Cu}(10 \mathrm{~nm})$ spacer to investigate STT-FMR in the nonlinear regime [20]. It was found that at high power levels of applied rf current the nanopillar behaves as a nonlinear forced oscillator whose frequency is a function of its amplitude.

Point contacts. Metallic point contacts are convenient tools for experimental studies of electron kinetics in metals [21-23]. High current densities associated with their extremely small size were instrumental for the first demonstration of STT in spin-valve multilayers [10] and for highfrequency magnetodynamics studies $[15,24,25]$. STT-FMR was demonstrated in mechanical point contacts to ultrathin films of exchanged bias spin valves (EBSV) [15]. A standard mechanical system $[10,23]$ can be used to establish an 
electrical contact between an electrochemically sharpened $\mathrm{Cu}$ tip and EBSV film (see Fig. 4(b)). Point contacts as small as a few nanometers in size were used to inject high densities of ac and dc currents into $\operatorname{IrMn}(10 \mathrm{~nm}) / \mathrm{NiFe}$ $(10 \mathrm{~nm}) / \mathrm{Cu}(5 \mathrm{~nm}) / \mathrm{NiFe}(4 \mathrm{~nm})$ spin valve, where the bottom (fixed) NiFe layer is exchange biased by the IrMn antiferromagnets [15]. The EBSV stack had a $30 \mathrm{~nm}$ thick $\mathrm{Cu}$ underlayer to produce a closely current-perpendicular-toplane (CPP) current flow across the spin valve.

Unlike most STT-FMR measurements made with strongly non-collinear free- and fixed-layer magnetizations, the point-contact experiment was performed in magnetic fields well above the saturation field of the spin valve with nearly collinear orientations of the two layers. Nevertheless, the resonance signal was clearly resolved (see Fig. 3) that may be associated with one of the symmetry breaking mechanisms discussed above. The latter, along with the simplicity of the mechanical point-contact technique extends the applicability of STT-FMR to smaller sample volumes, higher/lower frequencies, and a broader range of materials.

Electrodeposited nanowires. Very high current densities can be also created in magnetic nanowires produced by an electrochemical deposition into porous membrane templates. STT-FMR was successfully detected in single Co $(40 \mathrm{~nm}) / \mathrm{Cu}(5 \mathrm{~nm}) / \mathrm{Co}(5 \mathrm{~nm})[26]$ and $\mathrm{CoCu}(25 \mathrm{~nm}) / \mathrm{Cu}$ $(10 \mathrm{~nm}) / \mathrm{CoCu}(7 \mathrm{~nm}) / \mathrm{Cu}(10 \mathrm{~nm}) / \mathrm{CoCu}(100 \mathrm{~nm})$ [27] nanowires with diameters as small as $40 \mathrm{~nm}$. An rf current was injected into individual nanowires through a $\mathrm{Au}$ tip controlled by a differential screw mechanism similar to the point-contact setup discussed above. Rectified dc voltages corresponding to STT-FMR in both free and fixed layers were electrically detected. Interestingly, the nanowire device with three $\mathrm{CoCu}$ layers showed a resonance signal in zero applied magnetic field where STT-FMR presumably occurs in an effective field due to the magnetic coupling (e.g. dipolar) between the layers.

Time-resolved STT-FMR. While majority of STT-FMR experiments to date measure the dc (i.e., time independent) voltage response of a system, time resolved techniques were recently developed for detecting the time evolution of the magnetic precession driven by an ac currents [28]. The idea is to send a square-shaped pulse of radiofrequency current and use an oscilloscope to detect a time resolved voltage response after the falling edge of the pulse. The maximum amplitude of the response is determined by the spin torque of rf current while its decay reflects on the damping of precession. This time resolved technique has paved the way to a more accurate understanding of the STT-driven magnetodynamics in MTJs under high bias voltages and allowed to go beyond the lowest order approximation in the description of the bias dependence of STT torkances [28].

Alternative ways to generate STT-FMR. The driving force of magnetic precessions in STT-FMR is the oscillating STT torque associated with the ac spin current crossing a magnetic multilayer (see STT-FMR basics section). In all of the experiments discussed above the spin current is generated by driving an ac charge current across the multilayer. Recently, two alternative approaches were developed to generate ac spin current via spin Hall effect (SHE) [16] or spin diffusion [29].

In SHE-driven FMR experiments, a single ferromagnetic layer adjacent to a Pt layer (see Fig. 4(d)) can be excited into a uniform precession by a transverse spin current generated by a longitudinal charge current flowing in the $\mathrm{Pt}$ layer $[16,30]$. The resonance can be detected either (i) electrically by measuring a small rectified dc voltage, which appears at resonance when the longitudinal ac current mixes with the oscillating resistance of the magnet (controlled by AMR effect) [6,7], or (ii) by the Brillouin light scattering (BLS) technique [30] which probes the dynamic magnetization.

In STT-FMR experiments mediated by spin diffusion, the charge current is driven across a ferromagnetic/nonmagnetic $(\mathrm{F} / \mathrm{N})$ metal interface to produce a spin accumulation in the $\mathrm{N}$ metal [29]. A pure spin current then diffuses away from the interface and drives FMR in a free layer of a MTJ device positioned in direct proximity of the $\mathrm{F} / \mathrm{N}$ interface. The resonance is detected by measuring a rectified dc voltage which is a consequence of mixing between the oscillating MTJ resistance and an ac leakage current flowing through the MTJ.

\section{Summary}

The transfer of spin angular momentum from transport currents to local magnetic moments can drive the moments out of equilibrium. This STT effect provides a novel method to drive and characterize FMR in individual nanomagnets. The STT driven FMR has become a powerful tool to probe high-frequency magnetodynamics on the nanoscale. One of the direct outcomes of STT-FMR is the experimental determination of effective magnetic damping parameters in individual nanomagnets (including in the presence of electric current) with only a million of Bohr magnetons and scalable to even smaller dimensions. Due to the universality and scalability of STT-FMR as well as the versatility and high sensitivity of its electrical detection, this technique will no doubt continue contributing to our understanding of high-frequency magnetodynamics in magnetic nanostructures and spintronic devices. STT-FMR is instrumental not only for the understanding of fundamental physics involved in magnetodynamics on the nanoscale but also crucial to potential developments of magnetic memory and $\mathrm{rf}$ device applications.

This work was supported in part by NSF grants DMR0645377 and DMR-1207577. 
1. V.K. Arkad'yev, J. Russ. Phys. -Chem. Soc. 44, 165 (1912).

2. J.H.E. Griffiths, Nature 158, 670 (1946).

3. S.V. Vonsovskii, Ferromagnetic Resonance: The Phenomenon of Resonant Absorption of a High-Frequency Magnetic Field in Ferromagnetic Substances, Pergamon, Oxford (1966).

4. C. Kittel, Phys. Rev. 71, 270 (1947).

5. C. Kittel, Phys. Rev. 73, 155 (1948).

6. W.G. Egan and H.J. Juretschke, J. Appl. Phys. 34, 1477 (1963).

7. H.J. Juretschke, J. Appl. Phys. 31, 1401 (1960).

8. A. Yamaguchi, K. Motoi, A. Hirohata, H. Miyajima, Y. Miyashita, and Y. Sanada, Phys. Rev. B 78, 104401 (2008).

9. M. Harder, Z.X. Cao, Y.S. Gui, X.L. Fan, and C.-M. Hu, Phys. Rev. B 84, 054423 (2011).

10. J.C. Slonczewski, J. Magn. Magn. Mater. 159, 1 (1996).

11. L. Berger, Phys. Rev. B 54, 9353 (1996).

12. M. Tsoi, A.G.M. Jansen, J. Bass, W.C. Chiang, M. Seek, V. Tsoi, and P. Wyder, Phys. Rev. Lett. 804281 (1998).

13. A.A. Tulapurkar, Y. Suzuki, A. Fukushima, H. Kubota, H. Maehara, K. Tsunekawa, D.D. Djayaprawira, N. Watanabe, and S. Yuasa, Nature 438, 339 (2005).

14. J.C. Sankey, P.M. Braganca, A.G.F. Garcia, I.N. Krivorotov, R.A. Buhrman, and D.C. Ralph, Phys. Rev. Lett. 96, 227601(2006).

15. T. Staudacher and M. Tsoi, J. Appl. Phys. 109, 07C912 (2011)

16. L. Liu, Takahiro Moriyama, D.C. Ralph, and R.A. Buhrman, Phys. Rev. Lett. 106, 036601 (2011).

17. H. Kubota, A. Fukushima, K. Yakushiji, T. Nagahama, Sh. Yuasa, K. Ando, H. Maehara, Y. Nagamine, K. Tsunekawa,
D.D. Djayaprawira, N. Watanabe, and Y. Suzuki, Nature Physics 4, 37 (2008).

18. J.C. Sankey, Yong-Tao Cui, J.Z. Sun, J.C. Slonczewski, R.A. Buhrman, and Daniel C. Ralph, Nature Phys. 4, 67 (2008).

19. W. Chen, J.-M.L. Beaujour, G. de Loubens, A.D. Kent, and J.Z. Sun, Appl. Phys. Lett. 92, 012507 (2008).

20. W. Chen, G. de Loubens, J.-M.L. Beaujour, J.Z. Sun, and A.D. Kent, Appl. Phys. Lett. 95, 172513 (2009).

21. Y.V. Sharvin, Zh. Eksp. Teor. Fiz. 48, 984 (1965) [Sov. Phys. JETP 21, 655 (1965)].

22. A.G.M. Jansen, A.P. Van Gelder, and P. Wyder, J. Phys. C 13, 6073 (1980)

23. Y.G. Naidyuk and I.K. Yanson, Point-Contact Spectroscopy, Springer (2005).

24. O.P. Balkashin, V.V. Fisun, I.K. Yanson, L.Yu. Triputen, A. Konovalenko, and V. Korenivski, Phys. Rev. B 79, 092419 (2009).

25. Y. Okutomi, K. Miyake, M. Doi, H.N. Fuke, H. Iwasaki, and M. Sahashi, J. Appl. Phys. 109, 07 C727 (2011).

26. N. Biziere, E. Murè, and J.P. Ansermet, Phys. Rev. B 79, 012404 (2009).

27. A. Mourachkine, O.V. Yazyev, C. Ducati, and J.P. Ansermet, Nano Lett. 8, 3683 (2008).

28. C. Wang, Y.-T. Cui, J.A. Katine, R.A. Buhrman, and D.C. Ralph, Nature Phys. 7, 496 (2011).

29. L. Xue, C. Wang, Y.-T. Cui, L. Liu, A. Swander, J.Z. Sun, R.A. Buhrman, and D.C. Ralph, Phys. Rev. Lett. 108, 147201 (2012).

30. V.E. Demidov, S. Urazhdin, E.R.J. Edwards, and S.O. Demokritov, Appl. Phys. Lett. 99, 172501 (2011). 